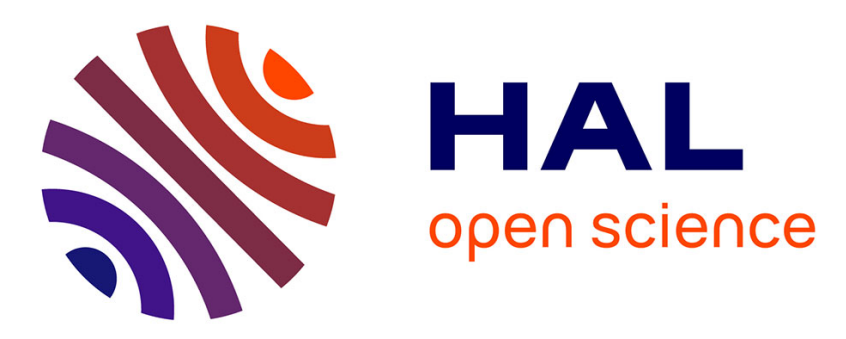

\title{
Reflecting Luneburg Lenses
}

Jorge Ruiz-Garcia, Enrica Martini, Cristian Della Giovampaola, David González-Ovejero, Stefano Maci

\section{To cite this version:}

Jorge Ruiz-Garcia, Enrica Martini, Cristian Della Giovampaola, David González-Ovejero, Stefano Maci. Reflecting Luneburg Lenses. IEEE Transactions on Antennas and Propagation, 2021, 69 (7), pp.3924-3935. 10.1109/TAP.2020.3044668 . hal-03137418

\section{HAL Id: hal-03137418 https://hal-univ-rennes1.archives-ouvertes.fr/hal-03137418}

Submitted on 10 Feb 2021

HAL is a multi-disciplinary open access archive for the deposit and dissemination of scientific research documents, whether they are published or not. The documents may come from teaching and research institutions in France or abroad, or from public or private research centers.
L'archive ouverte pluridisciplinaire HAL, est destinée au dépôt et à la diffusion de documents scientifiques de niveau recherche, publiés ou non, émanant des établissements d'enseignement et de recherche français ou étrangers, des laboratoires publics ou privés. 


\title{
Reflecting Luneburg Lenses
}

\author{
Jorge Ruiz-García, Enrica Martini, Senior Member, IEEE, Cristian Della Giovampaola, \\ David González-Ovejero, Senior Member, IEEE, and Stefano Maci, Fellow, IEEE
}

\begin{abstract}
This paper presents the exact closed-form solution for a new planar lens, hereinafter referred to as Reflecting Luneburg Lens (RLL). The proposed structure consists of two stacked parallel plate waveguides of circular shape. The rays generated by a point source located at the periphery of the bottom waveguide propagate along curvilinear paths, whose trajectories result from a variable refractive index profile with azimuthal symmetry. Then, these rays encounter a reflecting boundary and emerge all parallel in the upper uniform waveguide. The behavior of this lens resembles that of a flat Luneburg lens, with the fundamental difference that it works in reflection. The exact refractive index profile is found by solving the non-linear integral equation of ray-congruence trough a truncated Abel transform method. The concept is numerically verified through different implementations of the effective refractive index profile, including a metasurface-based implementation. The proposed lens triggers new possibilities that the normal flat Luneburg lens does not offer, and it is applicable in a large variety of microwave, terahertz and optical devices.
\end{abstract}

Index Terms-Beam-forming, flat optics, Luneburg lens, metasurface, surface wave.

\section{INTRODUCTION}

$\mathbf{L}$ UNEBURG graded index (GRIN) lenses [1] have been extensively used in the microwave frequency range [2], [3] and also at optical frequencies [4]. For instance, they constitute the only type of antenna capable of steering a beam in any direction without scan losses. On the other hand, planar Luneburg lenses in parallel plate waveguides (PPWs) provide full azimuthal scan with fan beams [5]. In this paper, we introduce a new flat lens that is also able to realize azimuthal scan, while opening up new possibilities like enabling the design of compact scanning antennas with an extended scanning range with respect to pillbox-based solutions [6], [7]. We denote it as Reflecting Luneburg Lens

Manuscript received July 09, 2020; revised xxxx.

The work of E. Martini and S. Maci was supported in part by the European Space Agency, under contract no. 4000127381/19/NL/AF “Antenna user terminal with wide angle impedance matching (WAIM) metamaterial radome", and in part by the Project of relevant Italian Interest (PRIN) "Metasurface Antennas for Satellite Applications" awarded by the Italian Ministry of University and Research. The work of J. Ruiz-García and D. González-Ovejero was funded in part by the European Union through the European Regional Development Fund (ERDF), and by the French Region of Brittany, Ministry of Higher Education and Research, Rennes Métropole and Conseil Départemental 35, through the CPER Project STIC \& Ondes. They also received the support of the Agence de l'Innovation de Défense (AID), the École des Docteurs de 1'UBL (Université Bretagne Loire) and the Conseil Régional de Bretagne.

J. Ruiz-García and D. González-Ovejero are with Univ Rennes, CNRS, Institut d'Électronique et de Télécommunications de Rennes (IETR), UMR 6164, 35000 Rennes, France.

E. Martini and S. Maci are with the Department of Information Engineering and Mathematics, University of Siena, 53100 Siena, Italy (e-mail: macis@dii.unisi.it).

C. Della Giovampaola is with Wave Up s.r.1., 53100 Siena, Italy.

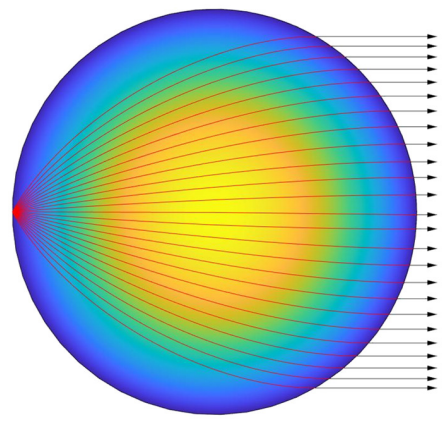

(a)

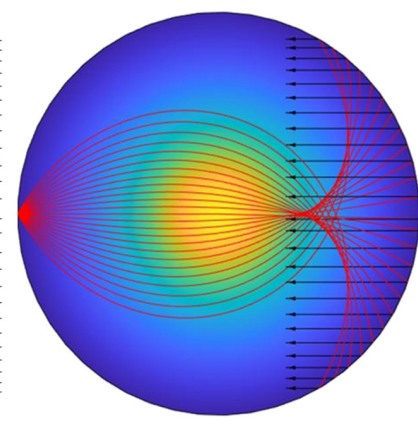

(b)
Fig. 1. Comparisons between (a) conventional Luneburg Lens and (b) Reflecting Luneburg Lens (RLL). In the RLL, the rays emerging from an arbitrary point at the periphery make longer paths and, after reflecting at the boundary, they are coupled to an upper uniform guiding layer, where they form a plane wave. The colored maps of refractive index do not have the same scale.

(RLL). Fig. 1 shows the difference between the ray-paths in a conventional flat Luneburg lens and in the RLL. The latter consists of two stacked PPWs, where the rays launched by a point source at the periphery of the bottom parallel plate (red lines in Fig. 1(b)) are first reflected on a metallic boundary, and then coupled to the upper uniform layer forming a planar wave-front (black lines in Fig. 1(b)).

The core of this article is the analytical derivation of the exact graded index profile for the RLL, which is obtained by a truncated Abel transform method. Despite the conceptual similarity with the Luneburg Lens, the expression obtained for the RLL graded index is very different and cannot be inferred from that of the conventional Luneburg lens. This fundamental difference is due to the longer ray path needed to match the reflection condition and the caustics inside the lens, shown in Fig. 1(b).

The RLL has never been studied before, notwithstanding its simplicity and practical usefulness. Indeed, this structure can be applied in a wide variety of optical, terahertz and microwave devices. The top PPW in the RLL can accommodate a radiating aperture to collimate the beam also in elevation, while preserving the lens total footprint. For instance, the top plate of the latter waveguide can be covered by a partially reflecting surface [8], [9], which can provide a pencil beam with conical scanning by changing the source position in the focal circumference. As an alternative, one can couple the RLL with a rotating prism, a linearly phased metasurface (MTS) or a continuous transverse stub (CTS) array to scan the beam also in elevation [10]-[13]. This way, it is possible to achieve $2 \mathrm{D}$ beam scanning with a number of control points that is only proportional to the perimeter of a flat structure in terms 
of free-space wavelengths, and not to its area in wavelength square. Therefore, the complexity and cost of the associated electronics are drastically reduced. These possibilities are not offered by the planar Luneburg lens.

Flat Luneburg lenses may be used to illuminate a MTS sinusoidally modulated along one direction [14] to obtain 2D scanning. In such configuration, the Luneburg lens and the MTS antenna are at the same level, so the total footprint of the antenna system is much larger than in the two-layered RLL described above. In addition, the ideal relative position between the lens and the MTS aperture depends on the position of the source. Hence, the extent of the radiating aperture in [14] had to be significantly increased to get multiple beams by accommodating several sources around the Luneburg lens. This led to an additional reduction of the aperture efficiency. There exist other compact alternatives, where two stacked PPWs have been used in combination with a parabolic reflector to generate a plane wave [6], [7]. However, the scanning performance of the parabolic reflecting structure degrades (deterioration in side-lobe levels arising from coma phase errors) when the source is displaced from the reflector focal point to steer the beam. One does not face such a limitation when scanning with the RLL.

In addition, the RLL is well suited to be implemented by MTSs, due to their intrinsic flatness. One can find in the open literature several articles in which the GRIN medium in conventional Luneburg lenses is implemented by modulated MTSs [15]-[19]. MTSs can manipulate the propagation of surface waves or guided waves in a similar way as graded index media, but they provide additional degrees of freedom in terms of design and simplify the technological implementation. Indeed, by averaging the boundary tangential fields, the MTS can be macroscopically described through homogenized impedance boundary conditions [20]. A gradual change in the dimensions of the MTS constituent elements leads to a spatial variability of the homogenized impedance boundary condition. This variation imposes a deformation of the surface wave or guided wave wavefront and addresses the local wavevector along non-rectilinear paths [21]-[26], just like in graded index media.

The propagation of surface waves and guided waves on modulated MTSs has been the subject of several recent works [15]-[19], [22]-[26], some of them addressing these phenomena in the framework of Transformation Optics (TO) [27]-[35] or in the framework of leaky-wave antennas [36][41]. A rigorous treatment concerning the control of surface waves with generic curvilinear wavefronts, denoted by flat optics, may be found in [42], where ray tracing, transport of energy, and ray velocity are rigorously described in terms of an equivalent refractive index for both isotropic and anisotropic impedance boundary conditions. This general theory is applied here to the particular case of an axially symmetric equivalent refractive index profile.

The article is organized as follows. Section II shows the derivation of the general analytical representation of the ray paths in a graded index medium with cylindrical symmetry to clarify the difference between Luneburg lenses and RLLs. This representation is exploited in Section III to derive the

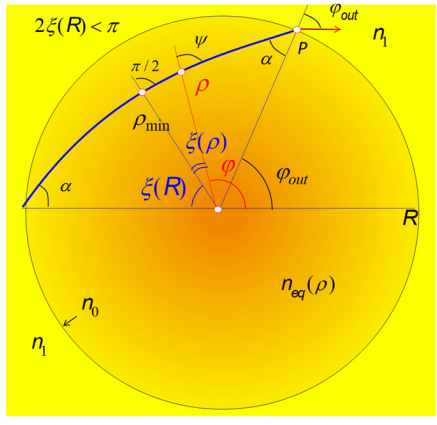

(a)

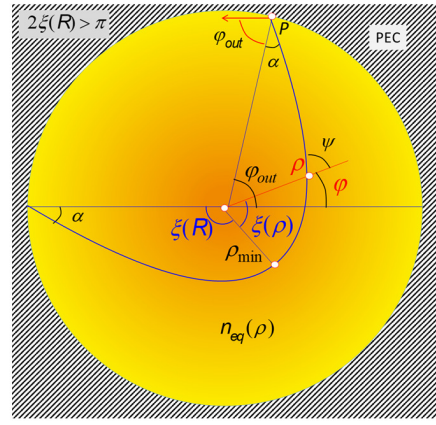

(b)
Fig. 2. Ray path in a PPW filled with a radially graded index medium for two cases: (a) $2 \xi(R)<\pi$, (b) $2 \xi(R)>\pi$. Case (a) represents the ray-path in a conventional Luneburg lens and case (b) represents the ray-path in a RLL, when a PEC wall is around the lens rim and reflected rays are canalized in an upper PPW with refractive index $n_{1}$.

closed-form, exact expression of the refractive index profile for the RLL. In Section IV, this refractive index profile is implemented through different approaches and numerically simulated to evaluate its performance, showing the expected behavior in all the cases. This section also presents an application example of RLLs in a multibeam antenna system. Finally, conclusions are drawn in Section V. The paper is enriched by a mathematical Appendix to provide more details about the truncated Abel transform formulation.

\section{Ray-Optics IN AXIAlly Symmetric RefRactive INDEX MEDIA}

In this analysis, we consider circular structures of radius $R$ and a Cartesian reference system with coordinates $(x, y, z)$ and unit vectors $\hat{\boldsymbol{x}}, \hat{\boldsymbol{y}}, \hat{\boldsymbol{z}}$. The coordinates of the corresponding cylindrical reference system are $(\rho, \varphi, z)$. Let us consider the case of a PPW filled by a medium with an inhomogeneous refractive index $n_{e q}(\rho)$ with azimuthal symmetry. Alternatively, the waveguide could consist of a perfectly conducting top wall and an impenetrable impedance boundary condition as bottom wall. The impedance in the latter case serves to realize an equivalent refractive index $n_{e q}(\rho)$ in the waveguide. When the equivalent refractive index has a radial dependence only, the solving differential equation is written as [43]

$$
\frac{1}{\rho} \frac{d}{d \ell}\left[n_{e q} \rho^{2} \frac{d \varphi}{d \ell}\right]=0
$$

where $d \ell$ is the elementary length along a curvilinear ray-path. With the exclusion of the point $\rho=0$, this equation states that the quantity $n_{e q} \rho^{2} d \varphi / d \ell$ is constant along the ray.

Defining as $\psi$ the angle that the ray direction forms with the radial direction, one has $\rho d \varphi / d \ell=\sin \psi$ and then

$$
n_{e q} \rho \sin \psi=\text { const }=L,
$$

which is the generalization of Snell's law for radially graded index media. It states the conservation along the ray of a quantity analogous to the mechanical angular momentum. Therefore, $L$ is called "ray angular momentum" and its value is preserved at any point of the ray-path.

\section{ACCEPTED MANUSCRIPT}




\section{A. Analytical Form for the Ray-Paths}

Since $d \rho / d \ell=\cos \psi$, one has $\rho d \varphi / d \rho=\tan \psi$, that through (2) gives

$$
\rho \frac{d \varphi}{d \rho}=\mp \frac{L}{\sqrt{\left(\rho n_{e q}(\rho)\right)^{2}-L^{2}}},
$$

where the upper/lower sign applies for $\psi<\frac{\pi}{2}, \psi>\frac{\pi}{2}$. The trajectories of all the rays in a graded-index medium with radial dependence of the refractive index profile satisfy (3). We can further specify the expression above for the case in which the source is placed at $\left(\rho_{0}, \varphi_{0}\right)=(R, \pi)$. The geometry for the problem at hand is depicted in Fig. 2, where $\alpha$ is the angle formed with the $x$-axis by the ray stemming from the source. For any $\alpha$, the trajectory passes through a point closest to the origin, whose radial coordinate is denoted as $\rho_{\text {min }}$, where the local ray is orthogonal to the radial direction and one has $\psi=\pi / 2$. This minimum radial distance can be calculated from $n_{e q}\left(\rho_{\min }\right) \rho_{\min }=L$. We would like to point out that $\rho_{\min }$ is the value of the radial distance that cancels out the square root in (3).

Since a ray cannot propagate when its momentum is larger than $L$, the ray undergoes at $\rho_{\min }$ a turning point. The angle $\psi$ is always $>\pi / 2$ before reaching the turning point, and it becomes $<\pi / 2$ when the ray goes beyond. At the turning point, the radial coordinate also inverts its monotonic behavior with respect to the azimuthal angle. Therefore, it is convenient to integrate (3) from $\rho_{\min }$ to $\rho$ defining the positive angle

$$
\xi(\rho)=\int_{\rho_{\min }}^{\rho} \frac{|L|}{\rho^{\prime} \sqrt{\left(\rho^{\prime} n_{e q}\left(\rho^{\prime}\right)\right)^{2}-L^{2}}} d \rho^{\prime},
$$

which is the angular deviation of the ray-path arc from the turning point, as shown in Fig 2. Since $L$ is constant along the ray path, it can be obtained from its initial value at the source point, i.e., $L=R n_{0} \sin \alpha$. Due to the symmetry of the trajectory about the turning point, the azimuthal angle covered by the ray-path arc until the observation point can be written as $\xi(R)+\operatorname{sgn}\left(\frac{\pi}{2}-\psi\right) \xi(\rho)$. Hence, $2 \xi(R)$ is the total angle spanned by the ray path.

Now, let us consider the case shown in Fig. 2(a), where $2 \xi(R)<\pi$. The function $\varphi(\rho)$, where $\varphi \in[0, \pi)$, is given by

$$
\varphi(\rho)=\pi-\left(\xi(R)+\operatorname{sgn}\left(\frac{\pi}{2}-\psi\right) \xi(\rho)\right) ; 2 \xi(R)<\pi .
$$

Equation (5) is the explicit functional equation in polar coordinates $(\rho, \varphi)$ of the ray trajectory of momentum $L$ for a given profile $n_{e q}(\rho)$. Consider now the case in Fig. 2(b), where $\alpha$ is negative and $2 \xi(R)>\pi$. In that case, for any $\varphi \in(-\pi, \pi)$ one has

$$
\varphi(\rho)=-\pi+\left(\xi(R)+\operatorname{sgn}\left(\frac{\pi}{2}-\psi\right) \xi(\rho)\right) ; \quad 2 \xi(R)>\pi .
$$

It is interesting to note that the ray-path for $\alpha$ positive can be obtained by just changing the sign in front of $\xi(R)+\operatorname{sgn}\left(\frac{\pi}{2}-\right.$ $\psi) \xi(\rho)$, owing to the symmetry of the ray-paths with respect to the $x$-axis. One can also observe that, due to the symmetry of the problem, the ray forms an angle $\alpha$ with respect to the normal to the circumference that limits the lens' area. That is, the ray ends its trajectory inside the lens with the same angle with which it is launched from the source. This property stems from the conservation of the angular momentum. Therefore, at the exit point $P$ the angle $\varphi$ assumes the values

$$
\varphi_{\text {out }}= \pm(\pi-2 \xi(R)) \text { for } 2 \xi(R) \lessgtr \pi .
$$

\section{B. Luneburg and Reflecting Luneburg Conditions}

The two situations of ray-paths in Fig. 2(a) $(2 \xi(R)<\pi)$ and Fig. 2(b) $(2 \xi(R)>\pi)$ are quite different concerning ray focusing. When $2 \xi(R)<\pi$, it is possible to have congruent rays leaving the lens parallel to the $x$-axis. This ray behavior is the one found in the conventional Luneburg lens. However, the transmission ray-congruence in Fig. 2(a) is not possible when $2 \xi(R)>\pi$. Conversely, the ray congruence for $2 \xi(R)>\pi$ can be obtained in reflection, by placing a cylindrical wall of perfect electric conductor (PEC) at the rim of the lens. This configuration is the one represented in Fig. 2(b) and corresponds to the RLL. The Snell's law applied at the rim of the lens implies $n_{0} \sin \alpha=n_{1} \sin \varphi_{\text {out }}$ for the configuration in transmission shown in Fig. 2(a). Interpreting the reflected rays in Fig. 2(b) as transmitted into an upper PPW with uniform refractive index $n_{1}$, the same expression also applies in reflection, but with a different bound concerning the raypath length. Therefore, using (4), one can write

$$
\begin{aligned}
& \pi-\arcsin \left(\frac{n_{0}}{n_{1}} \sin |\alpha|\right) \stackrel{L L}{=} \\
& 2 \int_{\rho_{\text {min }}}^{R} \frac{\sin |\alpha|}{\rho^{\prime} \sqrt{\left(\rho^{\prime} n_{e q} / R n_{0}\right)^{2}-\sin ^{2} \alpha}} d \rho^{\prime}, \\
& \pi+\arcsin \left(\frac{n_{0}}{n_{1}} \sin |\alpha|\right) \stackrel{R L L}{=} \\
& 2 \int_{\rho_{\text {min }}}^{R} \frac{\sin |\alpha|}{\rho^{\prime} \sqrt{\left(\rho^{\prime} n_{e q} / R n_{0}\right)^{2}-\sin ^{2} \alpha}} d \rho^{\prime},
\end{aligned}
$$

where (8) corresponds to the case of the conventional Luneburg lens, while (9) stands for the RLL. It is important to note that both conditions hold for the entire angular spectrum $|\alpha| \in\left(0, \frac{\pi}{2}\right)$. The refractive index solution in transmission exists, and gives for $n_{0}=n_{1}$ the well-known refractive index of the Luneburg lens $n_{e q}=n_{0}\left[2-(\rho / R)^{2}\right]^{1 / 2}$. The graded index profile solution of (9) provides the RLL behavior, and it is derived in the next section.

\section{Exact Solution FOR THE REFLECting LunEburG LENS}

The non-linear integral equation in (9) can be solved in closed-form for $n_{0}=n_{1}$ using the truncated Abel transform method. To this end, let us set $R=1$, bearing in mind that the radial distance will be de-normalized with respect to the radius in the final solution. To ease the notation, we define the following quantities: $N(\rho)=n_{e q}(\rho) \rho / n_{0}, N\left(\rho_{\text {min }}\right)=l$, and thus $N(1)=1, l=\sin \alpha, l \in(0,1)$. With this notation, (9) becomes

$$
\frac{\pi}{2}+\frac{1}{2} \arcsin (l)=\int_{\rho_{\text {min }}}^{1} \frac{l}{\rho \sqrt{(N(\rho))^{2}-l^{2}}} d \rho .
$$




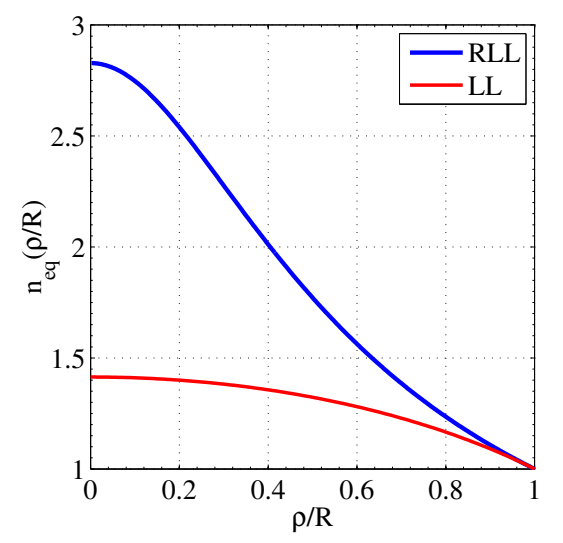

Fig. 3. Radial profile of the refractive index of the RLL (blue line) and of the equivalent Luneburg Lens (red line) for $n_{0}=n_{1}=1$.

Adopting in (10) the change of variable $x^{\prime}=\ln \rho$ and using the notation $\eta\left(x^{\prime}\right)=N\left(e^{x^{\prime}}\right)$ leads to

$$
\frac{\pi}{2}+\frac{1}{2} \arcsin (l)=\int_{\ln \rho_{\min }}^{0} \frac{l}{\sqrt{\eta^{2}\left(x^{\prime}\right)-l^{2}}} d x^{\prime} .
$$

The ray path equation in (11) is first manipulated by using the change of variables $\eta^{\prime}=\eta\left(x^{\prime}\right)$. Therefore, $x^{\prime}=x\left(\eta^{\prime}\right)$ and we can write (11) as

$$
\frac{1}{2} \pi+\frac{1}{2} \arcsin (l)=\int_{l}^{1} \frac{d x^{\prime}}{d \eta^{\prime}} \frac{l}{\sqrt{\eta^{\prime 2}-l^{2}}} d \eta^{\prime},
$$

where we have used $N(1)=1$ in the upper limit of the integral. Equation (12) shows that the function on the lefthand side is the inverse truncated Abel transform of the inverse function $x^{\prime}=x\left(\eta^{\prime}\right)$. Applying the inverse Abel transform [44] to both members leads to

$$
\frac{1}{2} \int_{\eta}^{1} \frac{\pi+\arcsin \left(l^{\prime}\right)}{\sqrt{l^{\prime 2}-\eta^{2}}} d l^{\prime}=-\frac{\pi}{2} \ln \rho .
$$

Due to the truncated upper limit, the above inversion formula cannot be easily demonstrated, and requires some algebraic steps done in Appendix A. Using the following identities

$$
\begin{aligned}
& \int_{\eta}^{1} \frac{\pi}{\sqrt{v^{2}-\eta^{2}}} d v=\pi \ln \left(\frac{1+\sqrt{1-\eta^{2}}}{\eta}\right), \\
& \int_{\eta}^{1} \frac{\arcsin (v)}{\sqrt{v^{2}-\eta^{2}}} d v=\frac{\pi}{2} \ln \left(1+\sqrt{1-\eta^{2}}\right),
\end{aligned}
$$

in (13) leads to

$$
-\ln \rho=\ln \left(\frac{1+\sqrt{1-\eta^{2}}}{\eta}\right)+\frac{1}{2} \ln \left(1+\sqrt{1-\eta^{2}}\right) .
$$

Considering that $\eta(\ln \rho)=N(\rho)=n_{e q}(\rho) \rho / n_{0}$, the inversion of (16), after re-normalizing by $R$, leads to

$$
n_{e q}=n_{0}\left(\frac{-1+\sqrt{1+8(\rho / R)^{2}}}{2(\rho / R)^{2}}\right)^{3 / 2},
$$

which is the exact form of the refractive index profile of the RLL. Despite the apparent similarity between (8) and

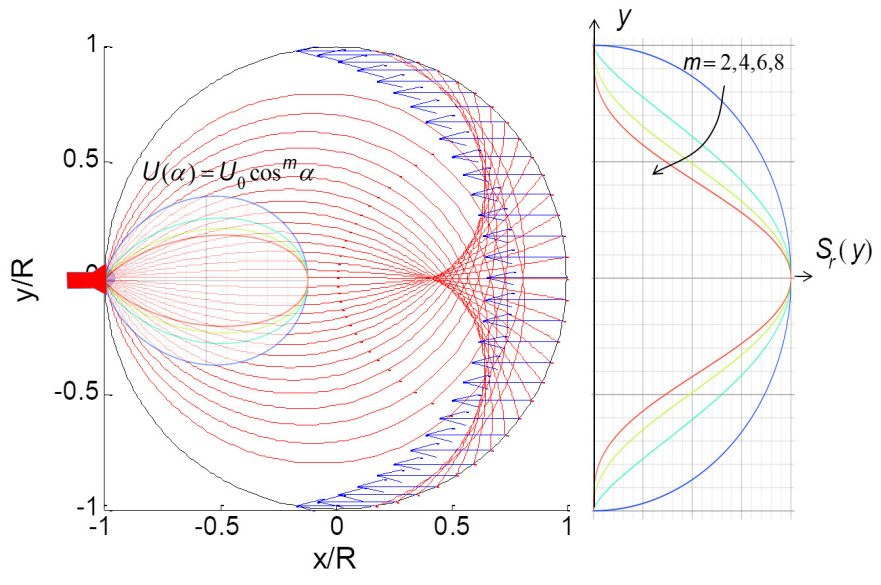

(a)

(b)

Fig. 4. (a) Ray paths for the RLL. The red and blue solid lines represent the rays in the bottom and top PPW, respectively. (b) Distribution of the energy density along $y$ for a feed with a power pattern of the type $U(\alpha)=$ $U_{0} \cos ^{m} \alpha$.

(9), the refractive index profile in (17) is quite different from that of the conventional Luneburg lens, which reads as $n_{e q}=n_{0}\left[2-(\rho / R)^{2}\right]^{1 / 2}$. The profile of the lens for $n_{0}=1$ is shown in Fig. 3. As one can observe in Fig. 3, the excursion range of the refractive index profile in the RLL is larger than in the Luneburg lens. Although this could be a disadvantage fabrication-wise, such refractive index values can be easily attained with the fully metallic metasurface structure described in Section IV-C.

\section{A. Closed Form of Ray-Paths and Fields}

To express the ray-paths in closed-form, we first solve for the turning point in $\rho_{\min } n_{e q}\left(\rho_{\min }\right) /\left(R n_{0}\right)=\sin \alpha$. Some algebraic steps yield the expression $\rho_{\min }=$ $R \sin |\alpha / 2| /\left[\sqrt{2} \cos ^{2}(\alpha / 2)\right]$. Then, using this explicit form for the turning point and (17), the ray-path in (4) can be rewritten as

$$
\xi(\rho)=\int_{\frac{\sin |\alpha / 2|}{\sqrt{2} \cos ^{2}(\alpha / 2)}}^{\rho / R} \frac{2 \sqrt{2} \sigma|\sin \alpha|}{\sqrt{\left(-1+\sqrt{\left.1+8 \sigma^{2}\right)^{3}-8 \sigma^{4} \sin ^{2} \alpha}\right.}} d \sigma
$$

where we have applied the substitution $\rho^{\prime} / R \rightarrow \sigma$. The ray paths for any $\alpha$ can then be found using (18) in (6). Fig. 4 shows the resulting ray-paths. The path lengths are the same as for the conventional Luneburg lens for $\alpha=0^{\circ}$, and increasingly longer for increasing $\alpha$. However, for $\alpha=50^{\circ}$, the ray path is just $30 \%$ longer than for the equivalent Luneburg lens. This additional propagation distance is necessary to collimate the reflected rays. Although in general this implies a small increase of the overall losses, this issue can be mitigated by metal-only implementations, as discussed in Section IV-C. On the other hand, if one places the primary feed in the inner focal region, the ray paths are shorter than for the equivalent Luneburg lens for $\alpha \in\left[-50^{\circ}, 50^{\circ}\right]$. Fig. 4 also shows that the two rays launched by the source in the vicinity of $\alpha= \pm \pi / 2$ 


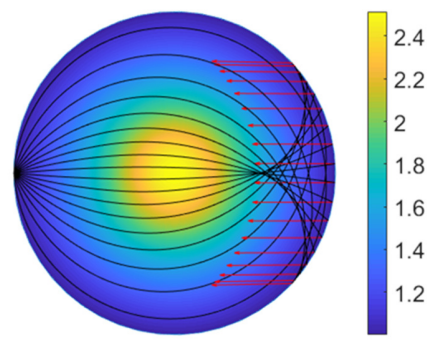

(a)

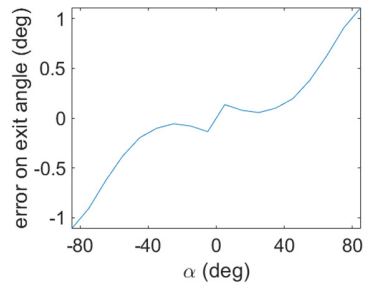

(b)
Fig. 5. (a) Ray-paths for a RLL with $\delta=0.7$. The black and red lines represent the rays in the bottom and top PPWs, respectively. (b) Difference between the exit angles obtained by the numerical solution of (19) and the approximate implicit formula in (21).

follow trajectories very close to the peripheral circumference and they contribute to an increase of energy density in the periphery of the planar wavefront, instead of being coupled to the upper parallel plate. It is therefore better to avoid launching rays in directions in the vicinity of $\alpha= \pm \pi / 2$. This can be simply done by using a primary source with a power pattern that damps the intensity of the rays as we approach $\alpha= \pm \pi / 2$, such as the $\cos ^{m} \alpha$ pattern represented in Fig. 4(b) for different values of $m$.

\section{B. Extension to Discontinuous Refractive Index at the Rim}

The explicit expression of the lens refractive index profile for different refractive indexes on top and bottom PPW, i.e., $n_{0} \neq n_{1}$, cannot be given in analytical form, but it is possible to find a very good approximation. The equation to be solved through the truncated Abel transform method is obtained by inserting $n_{0} / n_{1}$ in the argument of the arcsine function in (13) -as from (9)- and using (14). This leads to

$$
\pi \ln \left(\frac{1+\sqrt{1-\eta^{2}}}{\eta}\right)+\int_{\eta}^{1} \frac{\arcsin \left(\frac{n_{0}}{n_{1}} l^{\prime}\right)}{\sqrt{l^{\prime 2}-\eta^{2}}} d l^{\prime}=-\pi \ln \rho .
$$

The integral in (19) cannot be calculated in analytical form, but it can be approximated by expanding in Taylor series the arcsine function [45] and retaining the first two terms. After this manipulation, the integral can be estimated as

$$
\int_{\eta}^{1} \frac{\arcsin \left(\delta l^{\prime}\right)}{\sqrt{l^{\prime 2}-\eta^{2}}} d l^{\prime} \approx \sqrt{1-\eta^{2}}\left(\delta+\frac{\delta^{3}}{9}\left(\eta^{2}+\frac{1}{2}\right)\right) .
$$

Defining $\delta=n_{0} / n_{1}$, (20) yields an excellent approximation for $\delta \leq 0.7$. Substituting (20) in (19) leads to the following implicit expression for the RLL

$$
\begin{aligned}
& \pi \ln \left(\frac{1+\sqrt{1-\eta^{2}}}{\eta}\right)+ \\
& \sqrt{1-\eta^{2}}\left(\delta+\frac{\delta^{3}}{9}\left(\eta^{2}+\frac{1}{2}\right)\right)+\pi \ln \rho=0,
\end{aligned}
$$

where $\eta=\rho n_{e q} / n_{0}$. One can extend the range of applicability of the above formula by including more terms of the Taylor expansion in (20). Fig. 5(a) shows the ray-paths for a RLL with

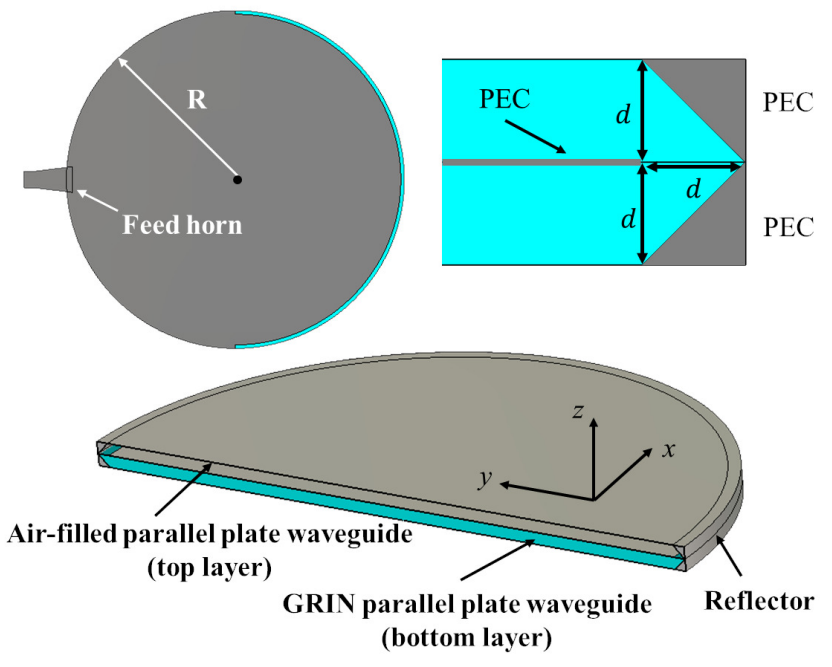

Fig. 6. Graded index TEM RLL with insets illustrating the corner reflector and the position of the feed horn.

$\delta=0.7$ in the bottom (black lines) and top (red arrows) PPWs, while the colormap represents the refractive index distribution. Fig. 5(b) shows the difference between the exit angles obtained by the numerical solution of (19) and by using (21). One can verify that the approximate formula yields an accuracy always better than one degree.

\section{Poynting Vector Profile in the Top PPW for Non-isotropic Feed Pattern}

Let us denote by $U(\alpha)$ the power density radiated by the source per unit angle and per unit thickness of the PPW. The conservation of the power density in each elementary ray tube gives $U(\alpha) d \alpha=S_{i}\left(\varphi_{\text {out }}\right) R d \varphi_{\text {out }}$, where $S_{i}(\varphi)$ represents the incident Poynting vector at $\varphi$. After the reflection at the periphery and coupling to the top PPW, the power density remains confined on a straight tube of flux along $x$. This condition leads to $S_{i}(\varphi) R d \varphi=S_{r}(y) d y$, where $S_{r}$ is the Poynting vector of the reflected ray and we have assumed that the top and bottom PPW have the same thickness. For $n_{0}=n_{1}$ one has $\alpha=\varphi_{\text {out }}=\arcsin (y / R)$, yielding

$$
S_{r}(y)=U \frac{d \alpha}{d \varphi_{\text {out }}} \frac{d \varphi_{\text {out }}}{d y}=\frac{U\left(\arcsin \frac{y}{R}\right)}{\sqrt{R^{2}-y^{2}}} .
$$

The equation above can be easily extended to $n_{0} \neq n_{1}$. It is seen that for uniform $U$ the density of reflected rays becomes infinite for $y=R$ and geometrical optics (GO) cannot be applied. However, (22) maintains its accuracy when the power density of the source is not uniform. Indeed, if the angular dependence of the source power density is of the type $U(\alpha)=$ $U_{0} \cos ^{m} \alpha$, (22) becomes

$$
S_{r}(y)=U_{0} \frac{\cos ^{m}\left(\arcsin \frac{y}{R}\right)}{\sqrt{R^{2}-y^{2}}},
$$

which leads to the distributions shown in Fig. 4(b) for $m=2$, 4,6 , and 8 . We note that for $m=1, S_{r}(y)$ becomes constant, so in order to have a uniform planar wavefront, one should have a $\cos \alpha$ pattern type of the feed. 


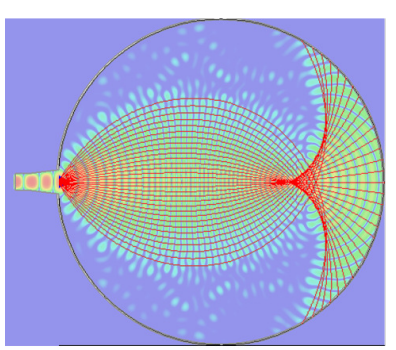

(a)

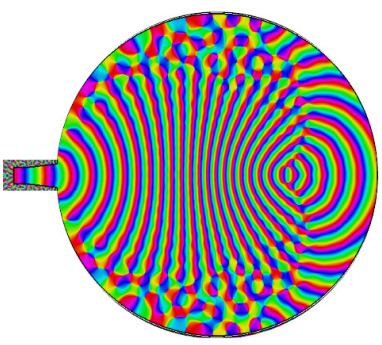

(c)

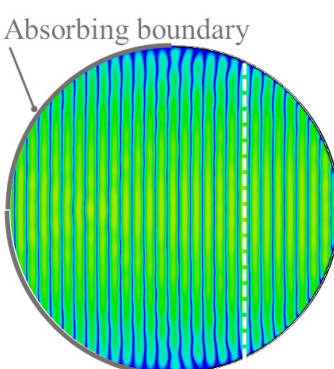

(b)

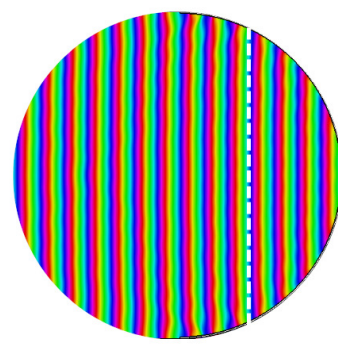

(d)
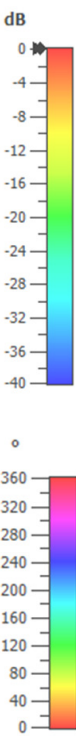

Fig. 7. Real part of the vertical component of the electric field in logarithmic scale for (a) the bottom and (b) the top PPWs in Fig. 6. Phase distribution in degrees for (c) the bottom and (d) the top PPWs in Fig. 6.

\section{Numerical Results}

\section{A. Graded Index Parallel Plate Lens}

In the first instance, to prove the validity of the closedform refractive index profile derived in Section III-A, we have analyzed an ideal RLL by a full-wave simulation. Fig. 6 shows the simulated structure. The structure consists of two stacked PPWs with circular shape. The bottom one is filled with a dielectric medium with the radially graded refractive index $n_{e q}(\rho)$ given in (17) and shown in Fig. 3. The top parallel plate is filled with air. Therefore, the refractive index in the top layer equals that at the rim of the bottom layer (Fig. 2(b)), such that $n_{0}=n_{1}=1$ in (9). A corner reflector is used to transfer the energy from the bottom to the top layer, and an absorbing boundary on the left half of this latter layer enables reflection-less plane-wave propagation. In this design, the frequency of operation is $f_{0}=30 \mathrm{GHz}$ and the radius of the lens is $R=7 \lambda_{0}$, where $\lambda_{0}$ is the free-space wavelength at $f_{0}$. This radius has been arbitrarily chosen for demonstration purposes. Reflecting Luneburg Lenses (RLLs) are intended for medium- and high-gain antennas, which means that radii $R \geq 2 \lambda_{0}$ are considered. The height $d$ of both PPWs is equal to $1.5 \mathrm{~mm}$, so only the fundamental transverse electromagnetic (TEM) mode propagates. The curvilinear TEM wavefronts are excited by a feed horn placed at $\left(\rho_{0}, \phi_{0}\right)=(R, \pi)$, and with a power density function approximated as $U(\alpha)=U_{0} \cos ^{m} \alpha$. The simulation results have been obtained with the commercial software CST Microwave Studio [46], since it allows one to use an ideal spatial GRIN material with a given profile. This feature enables a preliminary validation before carrying out an actual design based on MTSs.

Fig. 7(a) and Fig. 7(b) show the real part of the electric field's vertical component in the bottom and top PPWs,

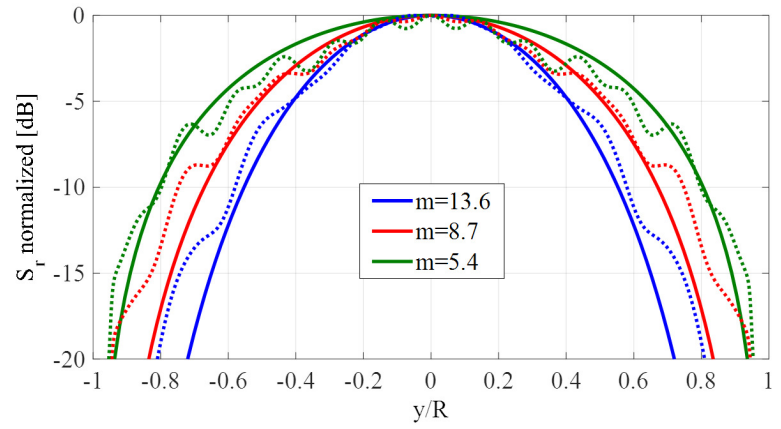

(a)

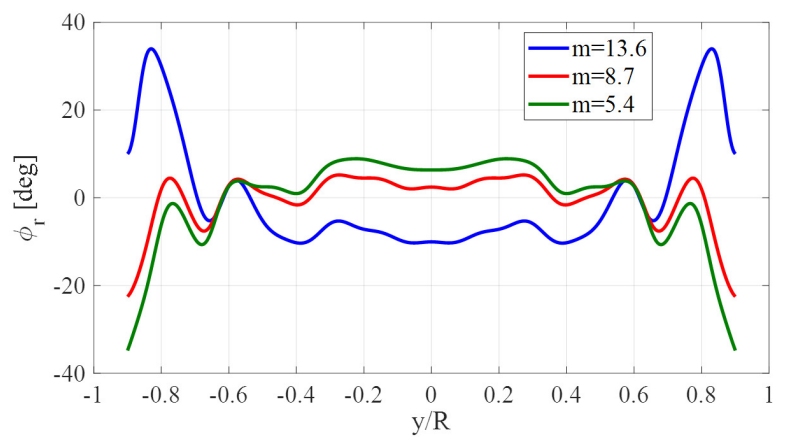

(b)

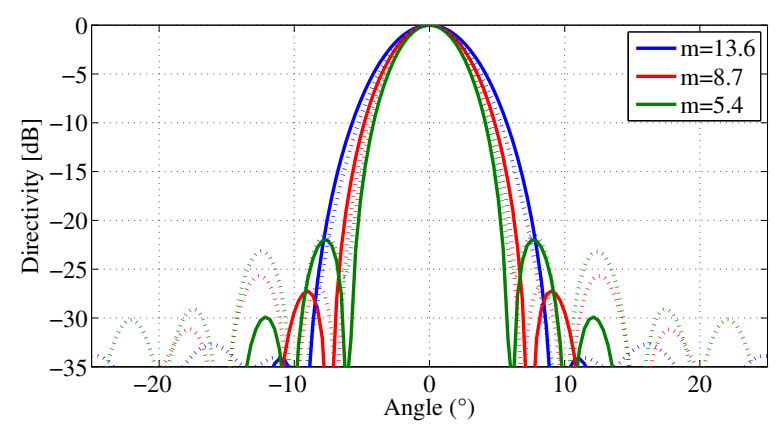

(c)

Fig. 8. (a) Power density distribution of the plane wave for sources with $m=5.4,8.7$, and 13.6. The dotted lines show the CST power density sampled in the top PPW along the white dashed line in Fig. 7(b), while the continuous lines have been calculated with (23). (b) Phase distributions for sources with $m=5.4,8.7$, and 13.6 computed with CST in the top PPW along the white dashed line in Fig. 7(d). (c) Calculated (solid lines) and simulated (dotted lines) far-field patterns corresponding to the power density distributions in (a).

respectively, and for a feed horn with $m=5.4$. In turn, Fig. 7(c) and (d) display the phase distributions obtained for the same component of the electric field in each layer. One can see that a plane wave is neatly generated in the top layer. The simulation also reveals that $91 \%$ of the accepted power is transmitted to this upper layer. Moreover, the wave-fronts given for the amplitude and phase distributions in Fig. 7(a) and Fig. 7(c) are, as expected, perpendicular to the ray paths, represented by red solid lines in Fig. 7(a).

Fig. 8(a) further illustrates the plane wave characteristics in the top PPW. The dotted lines represent the simulated normalized power density distribution of the plane wave in the top PPW along the white dashed line in Fig. 7(b) for $m=5.4,8.7$, and 13.6. The continuous lines have been 


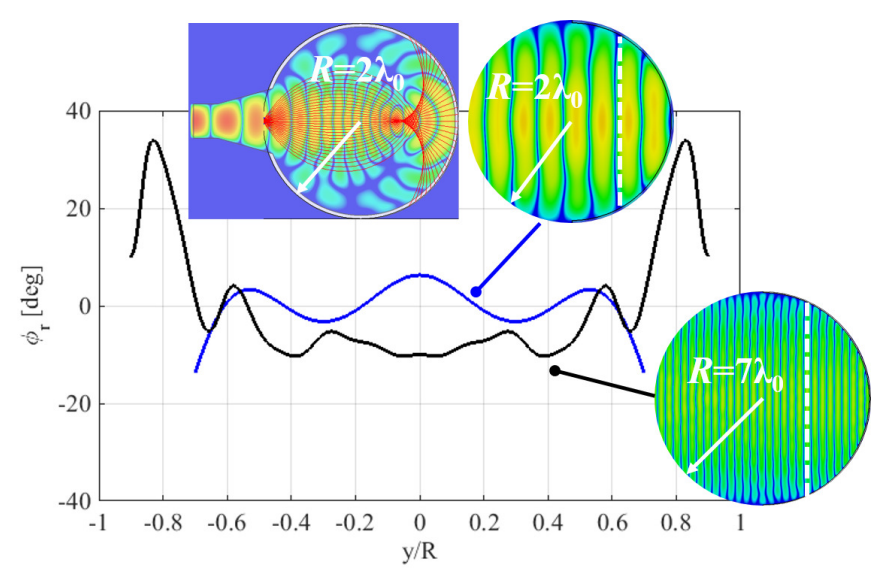

Fig. 9. Comparison between the phase distribution in the $7 \lambda_{0}$-radius lens in Fig. 8 (b) (black line) and the phase distribution in a $2 \lambda_{0}$-radius lens (blue line). The phases are calculated along the dashed lines in the insets. These insets show, for both lenses, the real part of the vertical component of the electric field ( $\left.\Re\left\{E_{z}\right\}\right)$ in the top parallel plates and in the bottom one for the $2 \lambda_{0}$-radius lens.

obtained using the analytical form in (23). The good agreement proves that one can also use the proposed analytical model to predict the power distribution in the RLL top layer. In turn, Fig. 8(b) shows the phase sampled along the white dashed line in Fig. 7(d) also for $m=5.4,8.7$, and 13.6. The far-field patterns obtained for the same values of $m$ with the analytical model (solid lines) and with the full-wave simulator (dotted lines) are shown in Fig. 8(c).

The RLL can also collimate the reflected beams in smaller apertures. As an example, Fig. 9 compares the phase distribution of the $7 \lambda_{0}$-radius lens in Fig. 8 with the phase distribution of a $2 \lambda_{0}$-radius lens, the primary feed is the same in both cases $(m=5.4)$. The insets show, for the two lenses, the real part of the vertical component of the electric field $\left(\Re\left\{E_{z}\right\}\right)$ in the top PPW and, for the $2 \lambda_{0}$-radius lens only, $\Re\left\{E_{z}\right\}$ in the bottom parallel plate. One can observe that a plane wave is

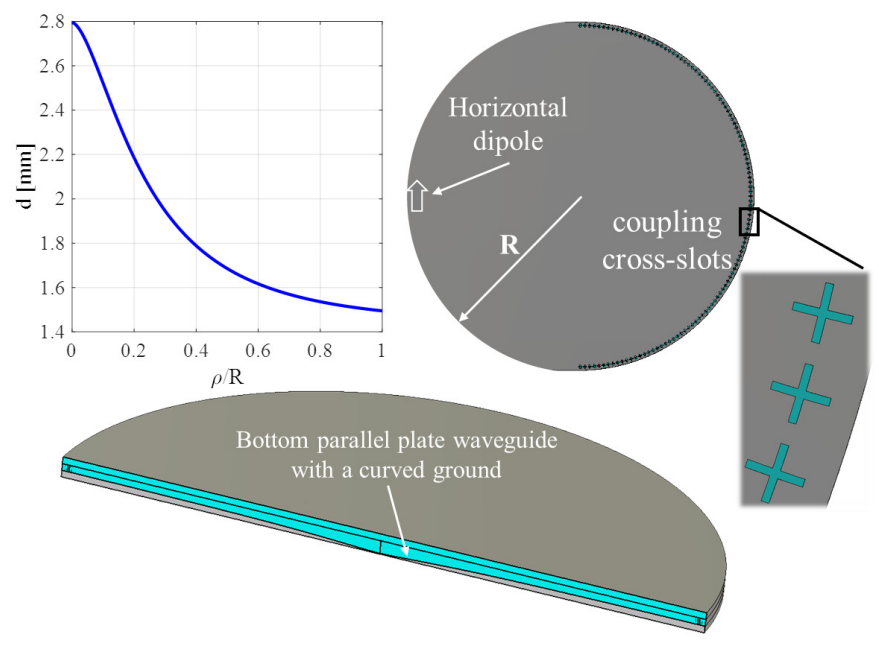

Fig. 10. Ground variable TE Reflecting Luneburg lens. The plot in the upper left corner shows the height variation of the bottom PPW with respect to the radius. The dielectric-filled top PPW is coupled by cross slots.

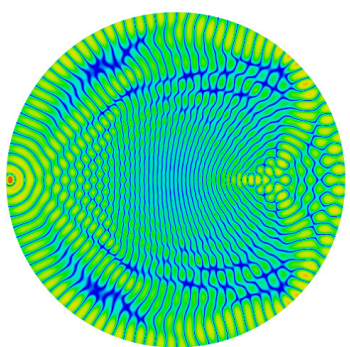

(a)

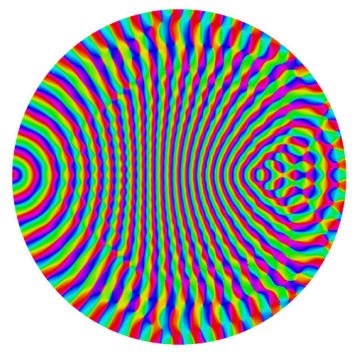

(c)

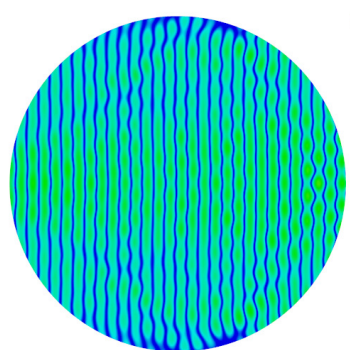

(b)

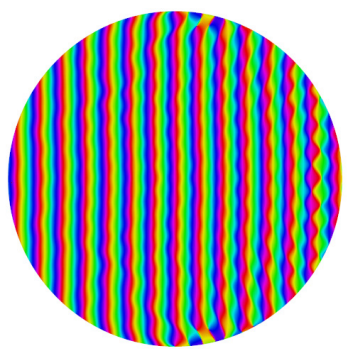

(d)

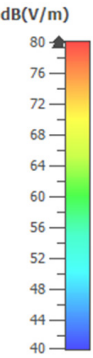

Fig. 11. Real part of the horizontal component of the electric field in logarithmic scale for (a) the bottom and (b) the top PPWs in Fig. 10. Phase distribution in degrees for (c) the bottom and (d) the top PPWs in Fig. 10.

well formed in the top PPW in both cases and that the flatness of the phase distributions is also very good.

\section{B. TE Parallel Plate Waveguide Lens}

In this second structure, the propagating mode is transverse electric (TE). The propagation constant of TE modes in a parallel plate waveguide is $\beta=\sqrt{\varepsilon_{r} k^{2}-(\pi / d)^{2}}$, where $d$ is the distance between the metal plates. Since $\beta$ and the refractive index are related by $n_{e q}=\beta / k$, one can obtain the desired profile by just changing the height of the bottom parallel plate waveguide. Fig. 10 shows the proposed structure, where the bottom parallel plate waveguide has a curved ground plane and it is filled by a homogeneous dielectric. Therefore, the local PPW height is obtained as $d(\rho)=\lambda_{0} /\left(2 \sqrt{\varepsilon_{r}-n_{e q}^{2}(\rho)}\right)$ [47], where $n_{e q}$ is given in (17). The inset in Fig. 10 shows the local height of the bottom parallel plate for a dielectric medium with relative permittivity $\varepsilon_{r}=12.2$. The lens radius is $R=7 \lambda_{0}$, where $\lambda_{0}$ is the free-space wavelength at $30 \mathrm{GHz}$ and the height $d$ of the upper waveguide is $1.5 \mathrm{~mm}$.

The CST simulation set-up is analogous to the one used in the previous example, although a different excitation is used. A horizontal dipole placed at the rim of the lens excites the first TE mode, while avoiding coupling power to the fundamental TEM mode. The modes of higher order than this TE are in cut-off. Moreover, cross slots (with arms 0.2-mm wide and 1.5$\mathrm{mm}$ long) are inserted in the middle metallic plate (shared by the top and bottom PPWs) at a distance $\lambda_{1} / 2$ from the border, where $\lambda_{1}$ is the effective wavelength. These slots, shown in the right inset of Fig. 10, serve to couple the TE mode to the upper PPW. The resulting horizontal E-fields in amplitude and phase are presented in Fig. 11 for both layers. The use of a less directional source leads to less clear wavefronts in the bottom

\section{ACCEPTED MANUSCRIPT}



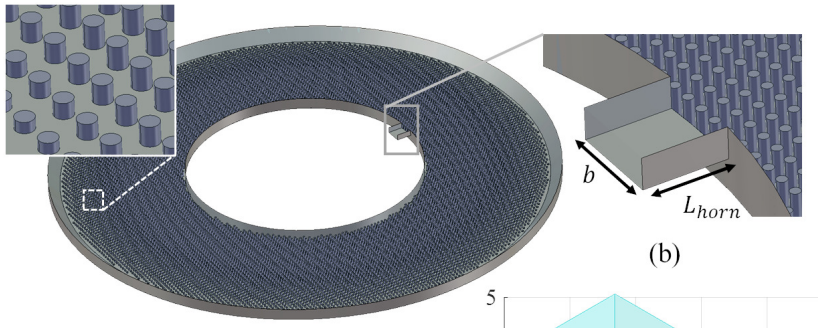

(b)

(a)

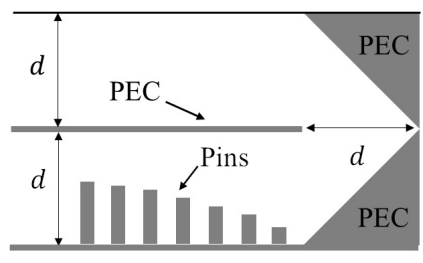

(c)

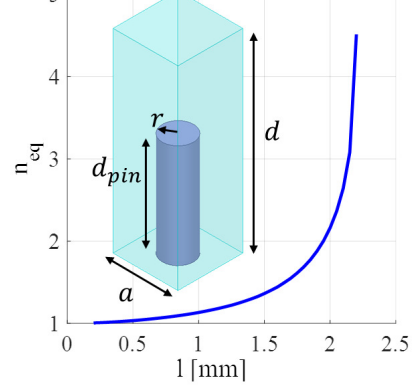

(d)

Fig. 12. TM Reflecting Luneburg lens implemented by a bed of nail lens. (a) Perspective view of the bottom layer. (b) Feed-horn dimensions $(b=5.4 \mathrm{~mm}$ and $L_{\text {horn }}=\lambda_{0} / 2$ ). (c) Schematic side view of the corner reflector used to excite the top waveguide. (d) Local equivalent refractive index as a function of the pin height (the pin dimensions are $r=0.3 \mathrm{~mm}$ and $a=1.25 \mathrm{~mm}$ ).

layer. It is interesting to note that despite the fact that the TEM mode can propagate, its contribution is not significant in the top layer. Indeed, the planar wavefront in the top PPW appears to be quite clean, as shown in Fig. 11(b) and Fig. 11(d).

\section{TM Bed of Nails Lens With Internal Focal Circle}

The last example shows two MTS implementations $(R=$ $7 \lambda_{0}$ and $R=2 \lambda_{0}$ ) of the RLL using a bed of nails. In this case, the propagating mode in the bottom PPW is transverse magnetic (TM), as discussed in [16], [17], [48]-[50]. A bed of nails realization eliminates the problems related to dielectric losses at higher frequencies. Indeed, although one could imagine high cost and complexity for the fabrication of such structure, the metal additive manufacturing in [50] constitutes a cost-effective approach to grow the metallic cylinders on an aluminum base-plate with the tolerance required in the millimeter-wave range. The AlSi10Mg alloys typically used in metal 3D printers provide good electrical properties and low losses. On the other hand, in the sub-THz range, one can use Deep Reactive Ion Etching (DRIE) to create the desired 3D pattern in a silicon wafer, which is then metalized by sputtering gold [51], [52]. Hence, the final structure inherits the good conductivity of $\mathrm{Au}\left(\sim 2 \times 10^{7} \mathrm{~S} / \mathrm{m}\right)$ in the sub-THz range.

Another fundamental difference with respect to the previous examples is that, instead of placing the source at the periphery, the horn aperture is placed at $\left(\rho_{0}, \phi_{0}\right)=(0.5 R, 0)$, with its phase center (located behind the aperture) lying at $\left(\rho_{0}, \phi_{0}\right)=(0.47 R, 0)$. Although the maximum concentration of rays occurs at approximately $\left(\rho_{0}, \phi_{0}\right)=(0.46 R, 0)$, the position of the phase center has been adjusted in the focal region to obtain a better collimation effect. This is common practice in spherical reflectors [53], where one has a focal region instead of a focal point, and with deployable parabolic

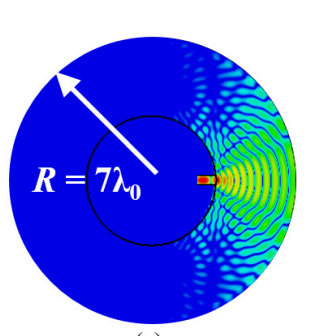

(a)

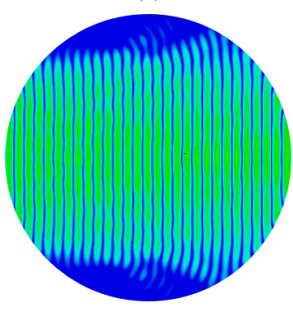

(c)

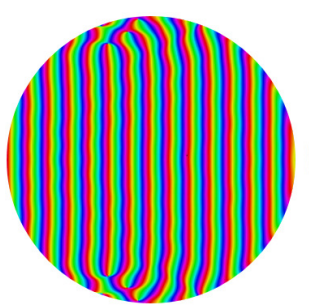

(e)

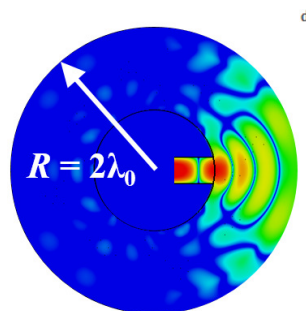

(b)

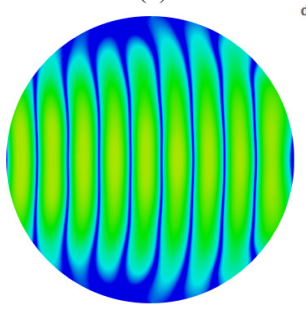

(d)

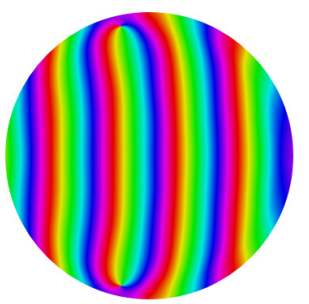

(f)

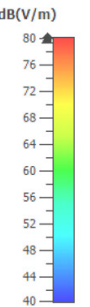

Fig. 13. Real part of the vertical component of the electric field $\left(\Re\left\{E_{z}\right\}\right)$ in logarithmic scale for the bottom PPWs loaded with pins in the (a) $R=7 \lambda_{0}$ and (b) $R=2 \lambda_{0}$ lenses. $\Re\left\{E_{z}\right\}$ in logarithmic scale for the top PPWs in the (c) $R=7 \lambda_{0}$ and (d) $R=2 \lambda_{0}$ lenses. Phase distribution in degrees for the top PPWs in the (e) $R=7 \lambda_{0}$ and (f) $R=2 \lambda_{0}$ lenses.

reflectors, where inaccuracies in the deployed surface also lead to a focal region [54]. The possibility of placing the source at the inner focal region is of utmost importance for practical applications, since the source and the corner reflector do not coexist in the focal circumference and it allows one to avoid undesired coupling. The height of the PPWs is $d=3.75 \mathrm{~mm}$, so the higher-order TM mode is in cut-off up to $40 \mathrm{GHz}$ [49]. The same corner reflector described in Section IV-A has been used to couple the TM mode power to the upper PPW.

Fig. 12(a) shows a perspective view of the resulting $R=$ $7 \lambda_{0}$ structure. The dimensions of the feed horn and the corner reflector are detailed in Fig. 12(b) and Fig. 12(c), respectively. The base-plate with the corner-reflector can be fabricated by standard lathe facing, boring and chamfering with $\pm 2 \mu \mathrm{m}$ roughness and a $\pm 5 \mu \mathrm{m}$ general tolerance. In this lens, the refractive index profile has been obtained varying the height of cylindrical pins arranged in a square lattice with unit-cell size $a=1.25 \mathrm{~mm}$ [16], [17], [50]. Fig. 12(d) shows the curve that relates the height of the pins to the equivalent refractive index $n_{e q}$. The height of the pin for each radial distance is computed by mapping the value of $n_{e q}$ given by (17) to a pin height.

Fig. 13 shows the simulation results of the fields in the two MTS lenses. As expected, placing the source at $\left(\rho_{0}, \phi_{0}\right)=$ $(0.5 R, 0)$ does not change significantly the behavior of the lens, and a plane wave is efficiently generated in the top PPW. Although some reflections are seen inside the lenses ring in

\section{ACCEPTED MANUSCRIPT}




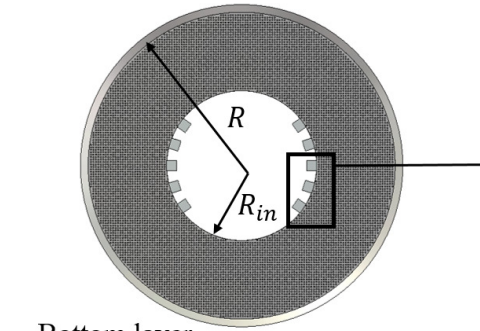

Bottom layer

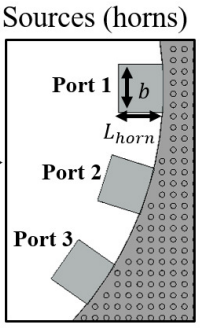

(b)

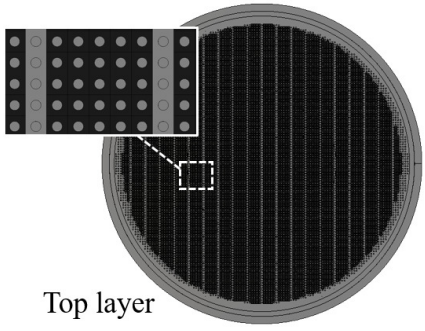

(c)

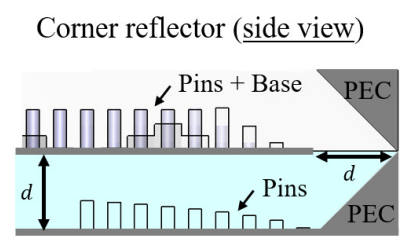

(d)
Fig. 14. TM Reflecting Luneburg lens as beam-former for a modulated MTS aperture. (a) Top view of the bottom layer with the multiple feeds. (b) Detail of the primary sources $\left(b=5.4 \mathrm{~mm}\right.$ and $\left.L_{\text {horn }}=\lambda_{0} / 2\right)$. (c) Top view of the modulated MTS aperture. (d) Side view of the corner reflector region.

Fig. 13(a) and Fig. 13(b), the power coupling efficiency in the top layer reaches $91 \%$ in both cases. Figs. 13(c)-(d) and Figs. 13(e)-(f) show the amplitude and phase distributions, respectively, of the plane-waves generated in the top PPWs.

\section{Example of antenna application}

From the symmetry of $n_{e q}(\rho)$, it is clear that the performance of the lens will be azimuthally symmetric. Therefore, different locations of the primary feed along $\phi$ will imply a rotation in the plane-wave direction. Here, we suggest a MTS antenna structure to scan the beam in both azimuth and elevation by combining source switching and mechanical rotation of the upper layer. The idea is to get an operation similar to that of Variable Inclination Continuous Transverse Stub (VICTS) array, where the beam is steered by changing the relative orientation of a planar wavefront and a slotted plate [12], [55], [56]. In the proposed structure, presented in Fig. 14, the RLL replaces the feeding network for the generation of the planar wavefront, while the role of the slotted plate is played by the periodically modulated MTS. The bottom layer is that of Fig. 12(a), where more ports have been placed. Moreover, the top layer is not a PPW anymore, but an open MTS structure sinusoidally modulated in one direction. The modulation is achieved by cylindrical pins placed over a stepped periodic ground plane, so the interface between the top of the pins and free-space is flat, as shown in Fig. 14(d). As in the previous section, the bottom PPW is connected with the upper one by a continuous corner reflector at the circular rim of the lens. The corner reflector in the top PPW is terminated by a circular flange that serves to excite the upper aperture, see Fig. 14(d). Since space is still available in the middle of the lower PPW after placing the primary sources (Fig. 14(a)-(b)), it is easy to arrange the top upper layer so that it can be rotated.
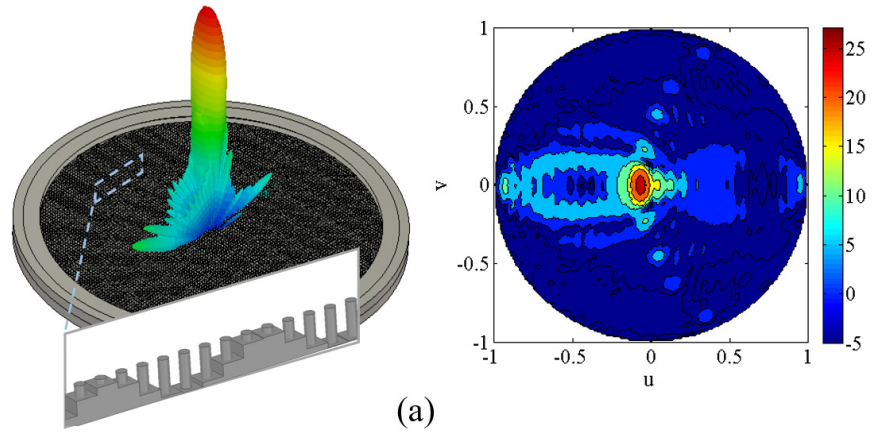

(a)
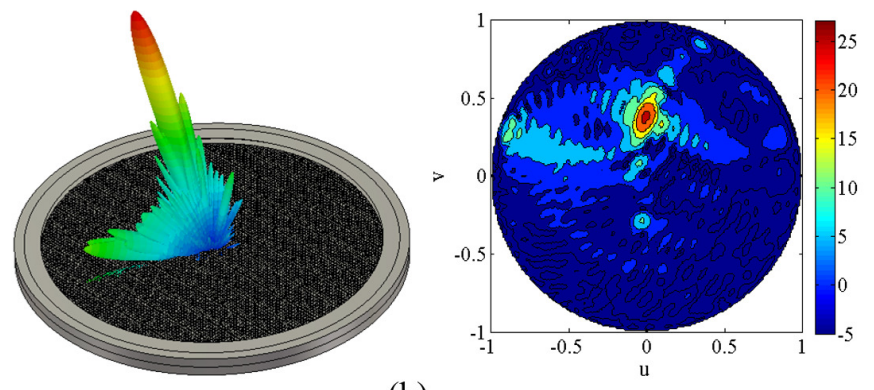

(b)
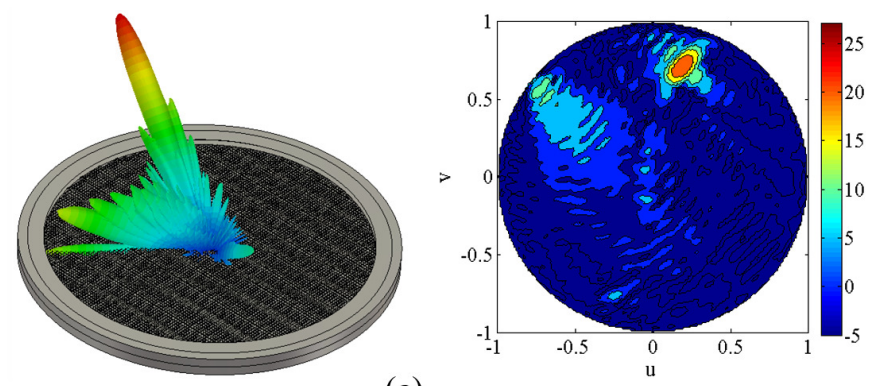

(c)

Fig. 15. 3D view of the radiated beam (left column) and u-v color maps (right column) showing the directivity in $\mathrm{dBi}$ when (a) port 1, (b) port 2 and (c) port 3 in Fig. 14(b) are excited.

Fig. 15 shows the radiation performance of the proposed antenna when three different ports are excited. The left-hand side figures schematically show the generated pencil beams when one independently excites port 1, port 2 and port 3 in Fig. 14(b). In turn, the right-hand side figures show the corresponding directivity in the $\mathrm{u}-\mathrm{v}$ plane. It is observed that when one switches ports the azimuth and elevation angles of the beam change. Notice that, as for the VICTS array, the relative angle between the port and the direction of sinusoidal modulation modifies both the azimuth and elevation angles of the radiated beam. Hence, one can cover a wide conical range by combining source switching with the rotation of the top layer with no need of rotating the bottom layer.

\section{CONCLUSiON}

We have presented the exact closed-form solution for the Reflecting Luneburg Lens and its implementation with metasurfaces. This new lens consists of two stacked parallel plate waveguides (PPW) of circular shape. The field generated by a primary source at an arbitrary point of the peripheral focal circumference or the inner focal region of the bottom PPW is collimated in the top uniform PPW, after reflection on

\section{ACCEPTED MANUSCRIPT}


a metallic boundary and coupling to the upper layer. The resulting structure is light weight, low profile and easy to manufacture, and it can be readily applied to beam-forming for antennas. In fact, after arranging an array of sources on the focal line or the inner focal region, one can obtain multiple simultaneous beams in azimuth or switch the beam direction by adding pin diodes. This means that it can be conveniently used as a beam-former for scanning antennas (without the need of phase shifters), when the upper layer is used as a radiating aperture.

\section{APPENDIX \\ DEMONSTRATION OF INVERSION FORMULA}

The steps followed to demonstrate the inversion formula in (13) are:

1) formally replace $l$ with $l^{\prime}$ in (12),

2) multiply both members by $1 / \sqrt{l^{\prime 2}-\eta^{2}}$,

3) integrate both members in $d l^{\prime}$ from $\eta$ to 1 .

With these steps we obtain

$$
\begin{aligned}
& \int_{\eta}^{1} \frac{\frac{1}{2} \pi+\frac{1}{2} \arcsin \left(l^{\prime}\right)}{\sqrt{l^{\prime 2}-\eta^{2}}} d l^{\prime}= \\
& \int_{\eta}^{1} \int_{l}^{1} \frac{d x^{\prime}}{d \eta^{\prime}} \frac{l^{\prime}}{\sqrt{l^{\prime 2}-\eta^{2}} \sqrt{\eta^{\prime 2}-l^{\prime 2}}} d l^{\prime} d \eta^{\prime} .
\end{aligned}
$$

We can interchange the order of integration in (24) applying Fubini's theorem. This implies that the extremes of integration can be defined only for positive value of the argument inside the square-roots, thus leading to

$$
\begin{aligned}
& \int_{\eta}^{1} \frac{\frac{1}{2} \pi \mp \frac{1}{2} \arcsin \left(l^{\prime}\right)}{\sqrt{l^{\prime 2}-\eta^{2}}} d l^{\prime}= \\
& \int_{\eta}^{1} \frac{d x^{\prime}}{d \eta^{\prime}}\left[\int_{\eta}^{\eta^{\prime}} \frac{l^{\prime}}{\sqrt{l^{\prime 2}-\eta^{2}} \sqrt{\eta^{\prime 2}-l^{\prime 2}}} d l^{\prime}\right] d \eta^{\prime} .
\end{aligned}
$$

One can recognize that

$$
\frac{l^{\prime}}{\sqrt{l^{\prime 2}-\eta^{2}} \sqrt{\eta^{\prime 2}-l^{\prime 2}}}=\left[\frac{d}{d l^{\prime}} \arcsin \left(\frac{\sqrt{l^{\prime 2}-\eta^{2}}}{\sqrt{\eta^{\prime 2}-\eta^{2}}}\right)\right],
$$

which inserted in (25) leads to

$$
\begin{aligned}
& \frac{1}{2} \int_{\eta}^{1} \frac{\pi-\arcsin \left(l^{\prime}\right)}{\sqrt{l^{\prime 2}-\eta^{2}}} d l^{\prime}= \\
& \int_{\eta}^{1} \frac{d x^{\prime}}{d \eta^{\prime}}\left[\arcsin \left(\frac{\sqrt{l^{\prime 2}-\eta^{2}}}{\sqrt{\eta^{\prime 2}-\eta^{2}}}\right)\right]_{\eta}^{\eta^{\prime}} d \eta^{\prime}
\end{aligned}
$$

The right-hand side can be rewritten as

$$
\int_{\eta}^{1} \frac{d x^{\prime}}{d \eta^{\prime}} \frac{\pi}{2} d \eta^{\prime}=\frac{\pi}{2} \int_{\ln \rho}^{0} d x^{\prime}=-\frac{\pi}{2} \ln \rho
$$

which leads to (13).

\section{REFERENCES}

[1] R. K. Luneburg, Mathematical Theory of Optics. CA, Berkeley: Univ. California Press, 1964.

[2] L. C. Gunderson and G. T. Holmes, "Microwave Luneburg lens," Appl. Opt., vol. 7, no. 5, pp. 801-804, May 1968.

[3] B. Fuchs, L. Le Coq, O. Lafond, S. Rondineau, and M. Himdi, "Design optimization of multishell Luneburg lenses," IEEE Trans. Antennas Propag., vol. 55, no. 2, pp. 283-289, Feb. 2007.

[4] Y.-Y. Zhao, Y.-L. Zhang, M.-L. Zheng, X.-Z. Dong, X.-M. Duan, and Z.-S. Zhao, "Three-dimensional Luneburg lens at optical frequencies," Laser \& Photonics Reviews, vol. 10, no. 4, pp. 665-672, Jun. 2016.

[5] Young-Jin Park, A. Herschlein, and W. Wiesbeck, "A photonic bandgap $(\mathrm{PBG})$ structure for guiding and suppressing surface waves in millimeterwave antennas," IEEE Trans. Microw. Theory Techn., vol. 49, no. 10, pp. 1854-1859, Oct. 2001.

[6] M. Ettorre, R. Sauleau, L. Le Coq, and F. Bodereau, "Single-folded leaky-wave antennas for automotive radars at $77 \mathrm{GHz}$," IEEE Antennas Wireless Propag. Lett., vol. 9, pp. 859-862, Sep. 2010.

[7] M. Ettorre, R. Sauleau, and L. Le Coq, "Multi-beam multi-layer leakywave SIW pillbox antenna for millimeter-wave applications," IEEE Trans. Antennas Propag., vol. 59, no. 4, pp. 1093-1100, Apr. 2011.

[8] K. A. Oyesina and A. M. H. Wong, "Metasurface-enabled cavity antenna: Beam steering with dramatically reduced fed elements," IEEE Antennas Wireless Propag. Lett., vol. 19, no. 4, pp. 616-620, Apr. 2020.

[9] A. H. Dorrah and G. V. Eleftheriades, "Peripherally excited phased array architecture for beam steering with reduced number of active elements," IEEE Trans. Antennas Propag., vol. 68, no. 3, pp. 1249-1260, Mar. 2020.

[10] G. F. Marshall, "Risley prism scan patterns," in Optical Scanning: Design and Application, L. Beiser, S. F. Sagan, and G. F. Marshall, Eds., vol. 3787, Int. Soc. Opt. Photon. SPIE, Jul. 1999, pp. 74-86.

[11] M. U. Afzal and K. P. Esselle, "Steering the beam of medium-tohigh gain antennas using near-field phase transformation," IEEE Trans. Antennas Propag., vol. 65, no. 4, pp. 1680-1690, 2017.

[12] W. Milroy, "The continuous transverse stub (CTS) array: basic theory, experiment, and application," in 1991 Antenna Applications Symposium, 1991.

[13] K. Tekkouk, J. Hirokawa, R. Sauleau, and M. Ando, "Wideband and large coverage continuous beam steering antenna in the $60-\mathrm{GHz}$ band," IEEE Trans. Antennas Propag., vol. 65, no. 9, pp. 4418-4426, 2017.

[14] Y. B. Li, R. Y. Wu, W. Wu, C. B. Shi, Q. Cheng, and T. J. Cui, "Dualphysics manipulation of electromagnetic waves by system-level design of metasurfaces to reach extreme control of radiation beams," Adv. Mater. Technol., vol. 2, no. 1, p. 1600196, 2017.

[15] C. Pfeiffer and A. Grbic, "A printed, broadband Luneburg lens antenna," IEEE Trans. Antennas Propag., vol. 58, no. 9, pp. 3055-3059, Sep. 2010.

[16] S. Maci, G. Minatti, M. Casaletti, and M. Bosiljevac, "Metasurfing: Addressing waves on impenetrable metasurfaces," IEEE Antennas Wireless Propag. Lett., vol. 10, pp. 1499-1502, Sep. 2011.

[17] M. Bosiljevac, M. Casaletti, F. Caminita, Z. Sipus, and S. Maci, "Non-uniform metasurface Luneburg lens antenna design," IEEE Trans. Antennas Propag., vol. 60, no. 9, pp. 4065-4073, Sep. 2012.

[18] J. A. Dockrey, M. J. Lockyear, S. J. Berry, S. A. R. Horsley, J. R. Sambles, and A. P. Hibbins, "Thin metamaterial Luneburg lens for surface waves," Phys. Rev. B, vol. 87, p. 125137, Mar. 2013.

[19] O. Quevedo-Teruel, J. Miao, M. Mattsson, A. Algaba-Brazalez, M. Johansson, and L. Manholm, "Glide-symmetric fully metallic Luneburg lens for 5G communications at Ka-band," IEEE Antennas Wireless Propag. Lett., vol. 17, no. 9, pp. 1588-1592, Sep. 2018.

[20] C. L. Holloway, E. F. Kuester, J. A. Gordon, J. O'Hara, J. Booth, and D. R. Smith, "An overview of the theory and applications of metasurfaces: The two-dimensional equivalents of metamaterials," IEEE Antennas Propag. Mag., vol. 54, no. 2, pp. 10-35, Apr. 2012.

[21] L. B. Felsen, "Evanescent waves," J. Opt. Soc. Am., vol. 66, no. 8, pp. 751-760, Aug. 1976.

[22] A. M. Patel and A. Grbic, "Modeling and analysis of printed-circuit tensor impedance surfaces," IEEE Trans. Antennas Propag., vol. 61, no. 1, pp. 211-220, Jan. 2013.

[23] M. Huang, S. Yang, F. Gao, R. Quarfoth, and D. Sievenpiper, "A 2-d multibeam half Maxwell fish-eye lens antenna using high impedance surfaces," IEEE Antennas Wireless Propag. Lett., vol. 13, pp. 365-368, 2014.

[24] R. Quarfoth and D. Sievenpiper, "Surface wave scattering reduction using beam shifters," IEEE Antennas Wireless Propag. Lett., vol. 13, pp. 963-966, 2014. 
[25] A. M. Patel and A. Grbic, "The effects of spatial dispersion on power flow along a printed-circuit tensor impedance surface," IEEE Trans. Antennas Propag., vol. 62, no. 3, pp. 1464-1469, Mar. 2014.

[26] M. Mencagli, E. Martini, and S. Maci, "Surface wave dispersion for anisotropic metasurfaces constituted by elliptical patches," IEEE Trans. Antennas Propag., vol. 63, no. 7, pp. 2992-3003, Jul. 2015.

[27] J. B. Pendry, D. Schurig, and D. R. Smith, "Controlling electromagnetic fields," Science, vol. 312, no. 5781, pp. 1780-1782, Jun. 2006.

[28] W. Tang, C. Argyropoulos, E. Kallos, W. Song, and Y. Hao, "Discrete coordinate transformation for designing all-dielectric flat antennas," IEEE Trans. Antennas Propag., vol. 58, no. 12, pp. 3795-3804, Dec. 2010.

[29] A. Vakil and N. Engheta, "Transformation optics using graphene," Science, vol. 332, no. 6035, pp. 1291-1294, Jun. 2011.

[30] R. Yang and Y. Hao, "An accurate control of the surface wave using transformation optics," Opt. Express, vol. 20, no. 9, pp. 9341-9350, Apr. 2012.

[31] A. M. Patel and A. Grbic, "Transformation electromagnetics devices based on printed-circuit tensor impedance surfaces," IEEE Trans. Microw. Theory Techn., vol. 62, no. 5, pp. 1102-1111, May 2014.

[32] E. Martini and S. Maci, Metasurface Transformation Theory. London: Springer London, 2014, pp. 83-116.

[33] M. jr Mencagli, E. Martini, D. González-Ovejero, and S. Maci, "Metasurface transformation optics," J. Opt., vol. 16, no. 12, p. 125106, Nov. 2014.

[34] M. Mencagli, E. Martini, D. González-Ovejero, and S. Maci, "Metasurfing by transformation electromagnetics," IEEE Antennas Wireless Propag. Lett., vol. 13, pp. 1767-1770, 2014.

[35] E. Martini, M. Mencagli, and S. Maci, "Metasurface transformation for surface wave control," Philosph. Trans. Royal. Soc. A Math. Phys. Eng. Sci., vol. 373, no. 2049, p. 20140355, Aug. 2015.

[36] B. H. Fong, J. S. Colburn, J. J. Ottusch, J. L. Visher, and D. F. Sievenpiper, "Scalar and tensor holographic artificial impedance surfaces," IEEE Trans. Antennas Propag., vol. 58, no. 10, pp. 3212-3221, Oct. 2010.

[37] A. M. Patel and A. Grbic, "A printed leaky-wave antenna based on a sinusoidally-modulated reactance surface," IEEE Trans. Antennas Propag., vol. 59, no. 6, pp. 2087-2096, Jun. 2011.

[38] G. Minatti, F. Caminita, M. Casaletti, and S. Maci, "Spiral leaky-wave antennas based on modulated surface impedance," IEEE Trans. Antennas Propag., vol. 59, no. 12, pp. 4436-4444, Dec. 2011.

[39] G. Minatti, S. Maci, P. De Vita, A. Freni, and M. Sabbadini, "A circularly-polarized isoflux antenna based on anisotropic metasurface," IEEE Trans. Antennas Propag., vol. 60, no. 11, pp. 4998-5009, Nov. 2012.

[40] G. Minatti, M. Faenzi, E. Martini, F. Caminita, P. De Vita, D. GonzálezOvejero, M. Sabbadini, and S. Maci, "Modulated metasurface antennas for space: Synthesis, analysis and realizations," IEEE Trans. Antennas Propag., vol. 63, no. 4, pp. 1288-1300, Apr. 2015.

[41] G. Minatti, F. Caminita, E. Martini, and S. Maci, "Flat optics for leakywaves on modulated metasurfaces: Adiabatic floquet-wave analysis," IEEE Trans. Antennas Propag., vol. 64, no. 9, pp. 3896-3906, Nov. 2016.

[42] E. Martini, M. Mencagli, D. González-Ovejero, and S. Maci, "Flat optics for surface waves," IEEE Trans. Antennas Propag., vol. 64, no. 1, pp. 155-166, Jan. 2016.

[43] M. Born, E. Wolf, A. B. Bhatia, P. C. Clemmow, D. Gabor, A. R. Stokes, A. M. Taylor, P. A. Wayman, and W. L. Wilcock, Principles of Optics: Electromagnetic Theory of Propagation, Interference and Diffraction of Light, 7th ed. Cambridge University Press, 1999.

[44] S. Cornbleet, "Geometrical optics reviewed: A new light on an old subject," Proc. IEEE, vol. 71, no. 4, pp. 471-502, Apr. 1983.

[45] E. Colombini, "Index-profile computation for the generalized Luneburg lens," J. Opt. Soc. Am., vol. 71, no. 11, pp. 1403-1405, Nov. 1981.

[46] CST of America, "CST Microwave Studio," Anaheim, CA, 2019.

[47] X. Wu and J. Laurin, "Fan-beam millimeter-wave antenna design based on the cylindrical luneberg lens," IEEE Trans. Antennas Propag., vol. 55, no. 8, pp. 2147-2156, Aug. 2007.

[48] M. G. Silveirinha, C. A. Fernandes, and J. R. Costa, "Electromagnetic characterization of textured surfaces formed by metallic pins," IEEE Trans. Antennas Propag., vol. 56, no. 2, pp. 405-415, Feb. 2008.

[49] A. Polemi, S. Maci, and P. Kildal, "Dispersion characteristics of a metamaterial-based parallel-plate ridge gap waveguide realized by bed of nails," IEEE Trans. Antennas Propag., vol. 59, no. 3, pp. 904-913, 2011.
[50] D. González-Ovejero, N. Chahat, R. Sauleau, G. Chattopadhyay, S. Maci, and M. Ettorre, "Additive manufactured metal-only modulated metasurface antennas," IEEE Trans. Antennas Propag., vol. 66, no. 11, pp. 6106-6114, Nov. 2018.

[51] D. González-Ovejero, C. Jung-Kubiak, M. Alonso-delPino, T. Reck, and G. Chattopadhyay, "Design, fabrication and testing of a modulated metasurface antenna at $300 \mathrm{GHz}$," in Proc. 11th Eur. Conf. Antennas Propag. (EUCAP), Paris, France, Mar. 19-24 2017, pp. 3416-3418.

[52] G. Chattopadhyay, T. Reck, C. Lee, and C. Jung-Kubiak, "Micromachined packaging for terahertz systems," Proc. IEEE, vol. 105, no. 6, pp. 1139-1150, 2017.

[53] R. Spencer and G. Hyde, "Studies of the focal region of a spherical reflector: Geometric optics," IEEE Trans. Antennas Propag., vol. 16, no. 3, pp. 317-324, 1968.

[54] N. Chahat, R. E. Hodges, J. Sauder, M. Thomson, E. Peral, and Y. Rahmat-Samii, "Cubesat deployable Ka-band mesh reflector antenna development for Earth science missions," IEEE. Trans. Antennas Propag., vol. 64, no. 6, pp. 2083-2093, 2016.

[55] A. Bhattacharyya, "Theory of beam scanning for slot array antenna excited by slow wave," IEEE Antennas Propag. Mag., vol. 57, no. 2, pp. 96-103, 2015.

[56] R. S. Hao, Y. J. Cheng, and Y. F. Wu, "Shared-aperture variable inclination continuous transverse stub antenna working at K- and Ka-bands for mobile satellite communication," IEEE Trans. Antennas Propag., vol. 68, no. 9, pp. 6656-6666, 2020. 